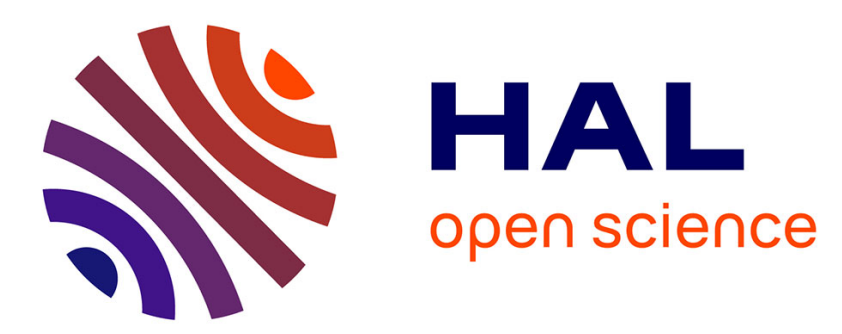

\title{
A Banach algebra approach to the weak spectral mapping theorem for locally compact abelian groups
}

\author{
Jean Esterle, Eva Fašangová
}

\section{To cite this version:}

Jean Esterle, Eva Fašangová. A Banach algebra approach to the weak spectral mapping theorem for locally compact abelian groups. Operator Theory: Advances and Applications, 2015, 250, pp.155-170. hal-00957211v2

\author{
HAL Id: hal-00957211 \\ https://hal.science/hal-00957211v2
}

Submitted on 13 Jul 2014

HAL is a multi-disciplinary open access archive for the deposit and dissemination of scientific research documents, whether they are published or not. The documents may come from teaching and research institutions in France or abroad, or from public or private research centers.
L'archive ouverte pluridisciplinaire HAL, est destinée au dépôt et à la diffusion de documents scientifiques de niveau recherche, publiés ou non, émanant des établissements d'enseignement et de recherche français ou étrangers, des laboratoires publics ou privés. 


\title{
A Banach algebra approach to the weak spectral mapping theorem for locally compact abelian groups
}

\author{
Jean Esterle and Eva Fašangová
}

\begin{abstract}
We give a general version of the weak spectral mapping theorem for non-quasianalytic representations of locally compact abelian groups which are weakly continuous in the sense of Arveson, based on a Banach algebra approach.

Mathematics Subject Classification (2000). Primary 47A16; Secondary 47D03, 46J40, 46H20.

Keywords. spectral mapping theorem, group representation, Arveson spectrum, infinitesimal generator, regular Banach algebra, Bochner integral.
\end{abstract}

\section{Introduction}

Let $\mathbf{T}=(T(t))_{t \in \mathbb{R}}$ be a strongly continuous one-parameter group of bounded operators on a Banach space $X$, and let $A$ be the infinitesimal generator of $\mathbf{T}$. Such a group is said to be non-quasianalytic if it satisfies the condition

$$
\sum_{n \in \mathbb{Z}} \frac{\log ^{+}\|T(n)\|}{1+n^{2}}<+\infty .
$$

This condition implies that the spectrum of $T(t)$ is contained in the unit circle. The weak spectral mapping theorem says that if the group is non-quasianalytic we have

$$
\overline{e^{t \sigma(A)}}=\sigma(T(t))
$$

The weak spectral mapping theorem in this form was stated by Marschall in 1986, [25], theorem 2.1-a, as a direct consequence of theorem 1.3 of [24] concerning decomposable operators and local multipliers. In the more general context of Banach modules and with the notion of Beurling spectrum, the weak spectral 
mapping theorem for representations of a group was stated by Baskakov in 1979, [3], lemma 3.

The theorem has been obtained independently and published by Lyubich and $\mathrm{Vu}$ in 1989 [35]. This paper is in fact a short note which shows how the theorem can be obtained as an easy consequence of results on "separability of the spectrum" of non-quasianalytic strongly bounded one-parameter groups proved by Lyubitch and Matsaev in 1962 in the seminal paper [21], which was later generalized by Lyubitch, Matsaev and Feldman [22], [23]. Finally, unaware of previous results, Nagel and Huang obtained again independently the weak spectral mapping theorem in [26]. Subsequently, Huang showed that the assumption of non-quasianalyticity is essential by giving an example in the quasianalytic case where the spectrum of the generator is empty [15], [16], which obviously prevents any form of spectral mapping theorem to hold.

Similar results can be obtained for more general groups of bounded operators on Banach spaces. Let $G$ be a locally compact abelian group, let $\mathbf{T}: g \mapsto T(g) \in$ $\mathcal{B}(X)$ be a representation of $G$ on a Banach space $X$, and assume that the representation is weakly continuous in Arveson's sense, see section 2. Set $\omega_{\mathbf{T}}(g)=\|T(g)\|$ for $g \in G$, denote by $\mathcal{M}_{\omega_{\mathrm{T}}}(G)$ the convolution algebra of all Borel measures $\mu$ on $G$ such that $\int_{G} \omega_{\mathbf{T}}(g) d|\mu|(g)<+\infty$. Also denote by $L_{\omega_{\mathbf{T}}}^{1}(G)$ the convolution algebra of all Haar measurable functions $f$ on $G$ such that $\int_{G}|f(g)| \omega_{\mathbf{T}}(g) d m(g)<+\infty$, where $m$ denotes the Haar measure on $G$. Then $L_{\omega_{\mathbf{T}}}^{1}(G)$ is an ideal of $\mathcal{M}_{\omega_{\mathbf{T}}}(G)$, and for $\mu \in \mathcal{M}_{\omega_{\mathrm{T}}}(G), x \in X$, the formula

$$
\phi_{\mathbf{T}}(\mu) x=\int_{G} T(g) x d \mu(g)
$$

defines an algebra homomorphism $\phi_{\mathbf{T}}: \mathcal{M}_{\omega_{\mathbf{T}}}(G) \rightarrow \mathcal{B}(X)$.

Now assume that $\lim _{n \rightarrow+\infty}\|T(n g)\|^{\frac{1}{n}}=1$ for every $g \in G$. Then $\omega_{\mathbf{T}}(g) \geq 1$ for $g \in G, \mathcal{M}_{\omega_{\mathbf{T}}}(G) \subset \mathcal{M}(G)$ and the Fourier transform $\mu \mapsto \widehat{\mu}$,

$$
\widehat{\mu}(\chi)=\int_{G}\langle g, \chi\rangle d \mu(g) \quad(\chi \in \widehat{G}),
$$

is well-defined on $\mathcal{M}_{\omega_{\mathbf{T}}}(G)$. The Arveson spectrum of $\mathbf{T}$ is defined by the formula

$$
\operatorname{spec}(\mathbf{T}):=\left\{\chi \in \widehat{G}: \hat{f}(\chi)=0 \forall f \in \operatorname{ker}\left(\phi_{\mathbf{T}}\right) \cap L_{\omega_{\mathbf{T}}}^{1}(G)\right\} .
$$

In section 5 we describe a well-known result, given in [10] or in proposition 3.18 of [9] in the case of bounded strongly continuous groups, which shows that if we identify $\widehat{\mathbb{R}}$ with $i \mathbb{R}$ then the spectrum $\sigma(A)$ of the generator of a one-parameter non-quasianalytic $C_{0}$-group $(T(t))_{t \in \mathbb{R}}$ equals the Arveson spectrum $\operatorname{spec}(\mathbf{T})$, and the weak spectral mapping theorem means that the set $\{\chi(t): \chi \in \operatorname{spec}(\mathbf{T})\}$ is dense in $\sigma(T(t))$ for every $t \in \mathbb{R}$.

The representation $\mathbf{T}$ is said to have the weak spectral mapping property if the set $\{\chi(g): \chi \in \operatorname{spec}(\mathbf{T})\}$ is dense in $\sigma(T(g))$ for every $g \in G$. In his celebrated paper on classification of type III factors [5], Connes shows that this is indeed the case for bounded representations (this result is stated in lemma 2.3.8 of [5] 
for unitary representations of locally compact abelian groups on Hilbert spaces, but the same argument works for bounded strongly continuous representations on general Banach spaces).

In the same direction d'Antoni, Longo and Zsidó observed in 1981 in [6] that if $\mathbf{T}: g \mapsto T(g)$ is a weakly continuous bounded representation of a locally compact abelian group on a Banach space, then $\left.\sigma\left(\phi_{\mathbf{T}}(f)\right)\right)=\widehat{f}(\operatorname{spec}(\mathbf{T})) \cup\{0\}$ for every $f \in L_{\omega_{\mathbf{T}}}^{1}(G)$.

The weak spectral mapping theorem means that if $\delta_{g}$ denotes the Dirac measure associated to $g \in G$, then the set $\widehat{\delta}_{g}(\operatorname{spec}(\mathbf{T}))$ is dense in $\sigma\left(\phi_{\mathbf{T}}\left(\delta_{g}\right)\right)$ for $g \in G$. Takahashi and Inoue showed in [33] that $\widehat{\mu}(\operatorname{spec}(\mathbf{T}))$ is dense in $\sigma\left(\phi_{\mathbf{T}}(\mu)\right)$ if $\mu$ is contained in the largest regular subalgebra $\mathcal{M}_{0}(G)$ of $\mathcal{M}(G)$ provided $\mathbf{T}$ is a weakly continuous bounded representation of a compact abelian group $G$, and Seferoğlu extended this result to locally compact abelian groups in [30] (see also his previous paper [29] for bounded one-parameter groups).

In the present paper we show in theorem 4.3 that, more generally, if $\mathbf{T}=$ $(T(g))_{g \in G}$ is a representation of a locally compact abelian group $G$ on $\mathcal{B}(X)$ which is weakly continuous in the sense of Arveson with respect to a dual pairing $\left(X, X_{*}\right)$ $\left(X_{*}\right.$ is a subspace of the dual space $X^{\prime}$ ) and satisfies the non-quasianalyticity condition

$$
\sum_{n=0}^{+\infty} \frac{\log \|T(n g)\|}{1+n^{2}}<+\infty \quad(g \in G)
$$

then $\widehat{\mu}(\operatorname{spec}(\mathbf{T}))$ is dense in $\sigma\left(\phi_{\mathbf{T}}(\mu)\right)$ for every measure $\mu \in \mathcal{M}_{\omega_{\mathbf{T}}}(G)$ which is contained in some closed regular subalgebra of $\mathcal{M}_{\omega_{\mathbf{T}}}(G)$, or, equivalently, which is contained in the largest closed regular subalgebra of $\mathcal{M}_{\omega_{\mathbf{T}}}(G)$. This result might be seen as well-known, but we could only find a reference for this in the case of oneparameter non-quasianalytic groups of bounded operators [31], in a slightly less general form: the result of [31] is stated for one-parameter $\mathcal{C}_{0}$-groups $(T(t))_{t \in \mathbb{R}}$ such that $\|T(t)\| \leq \omega(t)$ for some non-quasianalytic weight $w$, while our result is valid for all weakly continuous group representations where the weight $\omega_{\mathbf{T}}: g \mapsto\|T(g)\|$ is itself non-quasianalytic.

This paper offers also a Banach algebra approach of these spectral mapping theorems, which is a development of the methods used by the second author in [11], [12], [13]. In section 3 we give a general theorem concerning continuous unital homomorphisms $\phi: \mathcal{A} \rightarrow \mathcal{B}(X)$, where $\mathcal{A}$ is a commutative semisimple unital Banach algebra. Let $\mathcal{I}$ be a closed ideal of $\mathcal{A}$, and for $\chi \in \widehat{\mathcal{I}}$ (the Gelfand space) denote by $\tilde{\chi} \in \widehat{\mathcal{A}}$ the unique extension of $\chi$ to $\mathcal{A}$. Assume that $\mathcal{I}$ is a regular Banach algebra which satisfies spectral synthesis (see section 3 for the definition), and that we have

$$
\inf _{a \in \mathcal{I}}|\langle\phi(a) x-x, l\rangle|=0 \quad\left(x \in X, l \in X_{*}\right)
$$


for some dual pairing $\left(X, X_{*}\right)$ (see definition 2.1). We have for the hull (see section 3)

$$
h_{\mathcal{I}}(k e r(\phi) \cap \mathcal{I})=h_{\mathcal{I}}\left(k e r\left(\phi_{\mid \mathcal{I}}\right)\right)=\{\chi \in \widehat{\mathcal{I}}: \chi(u)=0 \forall u \in \operatorname{ker}(\phi) \cap \mathcal{I}\} \subset \widehat{\mathcal{I}} .
$$

We can summarize the spectral properties obtained in theorem 3.2 in the following table (in this table $\mathcal{U}$ denotes a closed regular subalgebra of $\mathcal{A}$ such that $\mathcal{I} \subset \mathcal{U}$, is the Gelfand transform, $\sim$ denotes the extension as above and $\operatorname{spec}(\phi(a))$ is the spectrum of the operator $\phi(a) \in \mathcal{B}(X))$.

\begin{tabular}{|c|c|c|}
\hline & $h_{\mathcal{I}}(\operatorname{ker}(\phi) \cap \mathcal{I})$ compact & $h_{\mathcal{I}}(k \operatorname{ker}(\phi) \cap \mathcal{I})$ noncompact \\
\hline$a \in \mathcal{A}$ & $\operatorname{spec}(\phi(a))=\widehat{a}\left(\tilde{h}_{\mathcal{I}}(\operatorname{ker}(\phi) \cap \mathcal{I})\right)$ & $\operatorname{spec}(\phi(a)) \subset \overline{\widehat{a}\left(\tilde{h}_{\mathcal{I}}(\operatorname{ker}(\phi) \cap \mathcal{I})\right)} \ni 0$ \\
$a \in \mathcal{U}$ & $\operatorname{spec}(\phi(a))=\widehat{a}\left(\tilde{h}_{\mathcal{I}}(\operatorname{ker}(\phi) \cap \mathcal{I})\right)$ & $\operatorname{spec}(\phi(a))=\widehat{\widehat{a}\left(\tilde{h}_{\mathcal{I}}(\operatorname{ker}(\phi) \cap \mathcal{I})\right)} \ni 0$ \\
$a \in \mathcal{I}$ & $\operatorname{spec}(\phi(a))=\widehat{a}\left(h_{\mathcal{I}}(\operatorname{ker}(\phi) \cap \mathcal{I})\right)$ & $\operatorname{spec}(\phi(a))=\widehat{a}\left(h_{\mathcal{I}}(k e r(\phi) \cap \mathcal{I})\right) \cup 0$ \\
\hline
\end{tabular}

Theorem 4.3 concerning non-quasianalytic weakly continuous representations of locally compact abelian groups is an application of theorem 3.2 in a concrete situation: $\mathcal{A}:=\mathcal{M}_{\omega_{\mathbf{T}}}(G), \phi:=\phi_{\mathbf{T}}, \mathcal{I}:=L_{\omega_{\mathbf{T}}}^{1}(G), \mathcal{U}:=$ a regular closed subalgebra of $\mathcal{M}_{\omega_{T}}(G)$. Note that in this context the Arveson spectrum $\operatorname{spec}(\mathbf{T})$ is actually $h_{L_{\omega_{\mathbf{T}}}^{1}(G)}\left(k \operatorname{er}\left(\phi_{\mathbf{T}}\right) \cap L_{\omega_{\mathbf{T}}}^{1}(G)\right)$.

The authors wish to thank Y. Tomilov for very valuable discussions concerning the history of the weak spectral mapping theorem and for pointing to our attention the references [22], [23], [24], [25], and to I. Kryshtal for drawing our attention to the works of A. G. Baskakov.

\section{Representations of locally compact abelian groups}

Let $X=(X,\|\|$.$) be a Banach space. We denote by \mathcal{B}(X)$ the Banach algebra of bounded linear operators $R: X \rightarrow X$ with composition, we denote by $\mathcal{G} \mathcal{L}(X)$ the group of invertible elements of $\mathcal{B}(X)$ and we denote by $I=I_{X}$ the identity map on $X$. We also denote by $\|$.$\| the operator norm on \mathcal{B}(X)$ associated to the given norm on $X$, and we denote by $\rho(R)$ the spectral radius of $R \in \mathcal{B}(X)$. If $Y$ is a subspace of the dual space $X^{\prime}$ of $X$ we will denote by $\sigma(X, Y)$ the weak topology on $X$ associated to $Y$.

We will use the following notion, introduced by Arveson in [2]. Notice that if we omit condition 2 we obtain the class of norming dual pairs used recently in [14], [20] to study Markov semigroups.

Definition 2.1. Let $X$ be a Banach space, and let $X_{*}$ be a subspace of the dual space $X^{\prime}$. We will say that $\left(X, X_{*}\right)$ is a dual pairing if the two following conditions are satisfied:

1. $\|x\|=\sup \left\{\langle x, l\rangle: l \in X_{*},\|l\| \leq 1\right\}$ for every $x \in X$. 
2. The $\sigma\left(X, X_{*}\right)$-closed convex hull of every $\sigma\left(X, X_{*}\right)$-compact subset of $X$ is $\sigma\left(X, X_{*}\right)$-compact.

For example, $\left(X, X^{\prime}\right)$ is a dual pairing. Also if $X=Y^{\prime}$ for some Banach space $Y$, and if we identify $Y$ with a subspace of $X^{\prime}=\left(Y^{\prime}\right)^{\prime}$ in the obvious way, then $(X, Y)$ is a dual pairing, see [2]. Notice that condition 1 means that if we set $\tilde{x}(l)=\langle x, l\rangle$ for $x \in X, l \in X_{*}$ then the map $x \mapsto \tilde{x}$ is an isometry from $X$ into the dual space $\left(X_{*}\right)^{\prime}$.

Let $S$ be a locally compact space, $u: S \rightarrow X$. Since $\|u(s)\|=\sup _{l \in X_{*}}|\langle u(s), l\rangle|$ for $s \in S$, the function $\omega_{u}: s \mapsto\|u(s)\|$ is lower semicontinuous on $S$, which allows to compute the upper integral

$$
\int_{S}^{*}\|u(s)\| d|\mu|(s):=\sup _{\substack{f \in \mathcal{C}_{c}^{+}(S) \\ f \leq \omega_{u}}} \int_{S} f(s) d|\mu|(s) \in[0,+\infty]
$$

for every regular measure $\mu$ on $S$, where $\mathcal{C}_{c}^{+}(S)$ denotes the space of all nonnegative compactly supported continuous functions on $S$.

Let $S$ be a locally compact space, and let $\left(X, X_{*}\right)$ be a dual pairing. A map $u: S \rightarrow X$ is said to be weakly continuous with respect to $\left(X, X_{*}\right)$ if the map $s \mapsto\langle u(s), l\rangle$ is continuous on $S$ for every $l \in X_{*}$, and we will often just say that $u$ is weakly continuous when no confusion may occur. In this situation it follows from the Banach-Steinhaus theorem and from condition 1 of definition 2.1 that we have, for every compact subset $K$ of $X$,

$$
\sup _{s \in K}\|u(s)\|=\sup _{s \in K}\|\widetilde{u(s)}\|=\sup _{s \in K} \sup _{l \in X_{*},\|l\| \leq 1}\langle u(s), l\rangle<+\infty .
$$

The following proposition is an immediate generalization of proposition 1.2 of [2].

Proposition 2.2. Let $S$ be a locally compact space, let $\left(X, X_{*}\right)$ be a dual pairing, and let $u: S \rightarrow X$ be a weakly continuous map. Set $\omega_{u}(s)=\|u(s)\|$ for $s \in S$, and denote by $\mathcal{M}_{\omega_{u}}(S)$ the set of all regular measures $\mu$ on $S$ such that $\|\mu\|_{\omega_{u}}:=$ $\int_{S}^{*}\|u(s)\| d|\mu|(s)<+\infty$. Then for every $\mu \in \mathcal{M}_{\omega_{u}}(S)$ there exists $x \in X$ satisfying

$$
\langle x, l\rangle=\int_{S}\langle u(s), l\rangle d \mu(s) \quad\left(l \in X_{*}\right) .
$$

Proof: Since $\int_{S}|\langle u(s), l\rangle| d|\mu|(s) \leq\|l\|\|\mu\|_{\omega_{u}}<+\infty$ for every $l \in X_{*}$, the formula $f_{\mu}(l):=\int_{S}\langle u(s), l\rangle d \mu(s)$ for $l \in X_{*}$ defines an element $f_{\mu} \in\left(X_{*}\right)^{\prime}$, and we have to show that $f_{\mu}=\tilde{x}$ for some $x \in X$. It follows from condition 1 of definition 2.1 that we have

$$
\left\|f_{\mu}\right\|=\sup _{l \in X_{*},\|l\| \leq 1}\left|\int_{S}\langle u(s), l\rangle d \mu(s)\right| \leq\|\mu\|_{\omega_{u}} .
$$

Denote by $\mathcal{M}_{c}(S)$ the space of all regular measures on $S$ supported by some compact subset of $S$. It follows from $(2.1)$ that $\mathcal{M}_{c}(S) \subset \mathcal{M}_{\omega_{u}}(S)$, and the fact that property $(2.2)$ holds for every $\mu \in \mathcal{M}_{c}(S)$ follows directly from [2], proposition 2.1. Set $\tilde{X}:=\{\tilde{x}: x \in X\}$. It follows from condition 1 of definition 2.1 that $\tilde{X}$ is 
closed in $\left(X_{*}\right)^{\prime}$. Let $\mu \in \mathcal{M}_{\omega_{u}}(S)$. There exists a sequence $\left(\mu_{n}\right)_{n \geq 1}$ of elements of $\mathcal{M}_{c}(S)$ such that $\lim _{n \rightarrow+\infty}\left\|\mu-\mu_{n}\right\|_{\omega_{u}}=0$. Hence $\lim _{n \rightarrow+\infty}\left\|f_{\mu}-f_{\mu_{n}}\right\|=0$, and $f_{\mu} \in \tilde{X}$.

When the conditions of proposition 2.2 are satisfied, we will use the notation

$$
x=\int_{S} u(s) d \mu(s),
$$

where the integral is a Pettis integral computed with respect to the $\sigma\left(X, X_{*}\right)$ topology, which defines an element of $X$ since $\left(X, X_{*}\right)$ is a dual pairing.

Notice that since $\omega_{u}$ is lower semicontinuous, it follows from the theory of integration on locally compact spaces, see [4], chapter 4 , that $\mathcal{M}_{\omega_{u}}(S)$ is the space of regular measures $\mu$ such that $\omega_{u}$ is integrable with respect to the total variation $|\mu|$ of $\mu$, and it follows from [4], proposition 1, that we have for $\mu \in \mathcal{M}_{\omega_{u}}(S)$

$$
\|\mu\|_{\omega_{u}}=\int_{S}\|u(s)\| d|\mu|(s)=\int_{S}^{*}\|u(s)\| d|\mu|(s)=\sup _{K \subset S, K \text { compact }} \int_{K}^{*}\|u(s)\| d|\mu|(s) .
$$

Let $G$ be a topological group. A representation of $G$ on a Banach space $X$ is a unital homomorphism $\mathbf{T}: G \rightarrow \mathcal{G L}(X)$, i.e. a map $g \mapsto T(g)$ satisfying $T\left(0_{G}\right)=I$ and $T\left(g_{1}+g_{2}\right)=T\left(g_{1}\right) T\left(g_{2}\right)$ for $g_{1}, g_{2} \in G$. We now introduce the notion of weakly continuous representation.

Definition 2.3. Let $G$ be a locally compact abelian group and let $X$ be a Banach space. A representation $\mathbf{T}$ of $G$ on $X$ is said to be weakly continuous with respect to a dual pairing $\left(X, X_{*}\right)$ if the map $g \mapsto\langle T(g) x, l\rangle$ is continuous on $G$ for every $x \in X$ and every $l \in X_{*}$. $G$ on $X$

We will often write $\mathbf{T}=(T(g))_{g \in G}$ when $\mathbf{T}: g \mapsto T(g)$ is a representation of

Let $\left(X, X_{*}\right)$ be a dual pairing, and let $\mathbf{T}=(T(g))_{g \in G}$ be a weakly continuous representation. We have, for $g \in G$,

$$
\|T(g)\|=\sup \left\{\langle T(g) x, l\rangle: x \in X,\|x\| \leq 1, l \in X_{*},\|l\| \leq 1\right\}
$$

and so the weight $\omega_{\mathbf{T}}: g \mapsto\|T(g)\|$ is lower semicontinuous on $G$. Let $K$ be a compact subset of $G$. Since $\sup _{g \in K}\|T(g) x\|<+\infty$ for every $x \in X$, it follows again from the Banach-Steinhaus theorem that $\sup _{g \in K}\|T(g)\|<+\infty$. In this situation we can define the weighted space $\mathcal{M}_{\omega_{\mathbf{T}}}(G)$ consisting of all regular measures $\mu$ on $G$ such that the upper integral $\int_{G}^{*}\|T(g)\| d|\mu|(g)$ is finite. Since $\omega_{\mathbf{T}}\left(g_{1}+g_{2}\right) \leq \omega_{\mathbf{T}}\left(g_{1}\right) \omega_{\mathbf{T}}\left(g_{2}\right)$ for $g_{1}, g_{2} \in G$, it follows from [4], Chap. 8, Prop. 2 that the convolution product $\mu * \nu$ is well-defined and belongs to $\mathcal{M}_{\omega_{\mathbf{T}}}(G)$ for $\mu, \nu \in \mathcal{M}_{\omega_{\mathbf{T}}}(G)$ and that $\left(\mathcal{M}_{\omega_{\mathbf{T}}}(G),\|\cdot\|_{\omega_{\mathbf{T}}}\right)$ is a Banach algebra with respect to convolution which contains the convolution algebra $\mathcal{M}_{c}(G)$ of compactly supported regular measures on $G$ as a dense subalgebra.

Denote by $L_{\omega_{\mathrm{T}}}^{1}(G)$ the convolution algebra of all Haar-measurable (classes of) functions $f$ on $G$ such that $f \omega_{\mathbf{T}}$ is integrable with respect to the Haar measure $m$ 
on $G$, identified with the space of all measures $\mu \in \mathcal{M}_{\omega_{\mathbf{T}}}(G)$ which are absolutely continuous with respect to the Haar measure.

The following result is an easy extension of [2], proposition 1.4.

Proposition 2.4. Let $G$ be a locally compact abelian group, let $\left(X, X_{*}\right)$ be a dual pairing, and let $\mathbf{T}=\left(T(g)_{g \in G}\right.$ be a weakly continuous representation of $G$ on $X$. The formula

$$
\phi_{\mathbf{T}}(\mu) x=\int_{G} T(g) x d \mu(g)
$$

defines for every $x \in X$ and every $\mu \in \mathcal{M}_{\omega_{\mathbf{T}}}(G)$ an element of $X, \phi_{\mathbf{T}}(\mu) \in \mathcal{B}(X)$ for every $\mu \in \mathcal{M}_{\omega_{\mathbf{T}}}(G)$, and $\phi_{\mathbf{T}}: \mu \mapsto \phi_{\mathbf{T}}(\mu)$ is a norm-decreasing unital algebra homomorphism from the convolution algebra $\mathcal{M}_{\omega_{\mathbf{T}}}(G)$ into $\mathcal{B}(X)$.

Moreover we have, for $x \in X, l \in X_{*}$,

$$
\inf _{f \in L_{\omega_{\mathbf{T}}}^{1}(G)}\left|\left\langle\phi_{\mathbf{T}}(f) x-x, l\right\rangle\right|=0 .
$$

Proof: Since $\|T(g) x\| \leq\|T(g)\|\|x\|$, the fact that formula (2.4) defines an element of $X$ for $x \in X$ and $\mu \in \mathcal{M}_{\omega_{\mathbf{T}}}(G)$ follows directly from proposition 2.2. We have

$$
\begin{aligned}
\left\|\phi_{\mathbf{T}}(\mu) x\right\| & =\sup _{l \in X_{*},\|l\| \leq 1}\left|\int_{G}\langle T(g) x, l\rangle d \mu(g)\right| \\
& \leq \sup _{l \in X_{*},\|l\| \leq 1} \int_{G}|\langle T(g) x, l\rangle| d|\mu|(g) \leq\|x\|\|\mu\|_{\omega_{\mathbf{T}}},
\end{aligned}
$$

and so $\phi_{\mathbf{T}}(\mu) \in \mathcal{B}(X)$ for $\mu \in \mathcal{M}_{\omega_{\mathbf{T}}}(G)$ and $\left\|\phi_{\mathbf{T}}(\mu)\right\| \leq\|\mu\|_{\omega_{\mathbf{T}}}$. As observed in [2], a routine application of Fubini's theorem shows that $\phi_{\mathbf{T}}(\mu * \nu)=\phi_{\mathbf{T}}(\mu) \phi_{\mathbf{T}}(\nu)$ for $\mu, \nu \in \mathcal{M}_{c}(G)$. Since $\mathcal{M}_{c}(G)$ is dense in $\mathcal{M}_{\omega_{\mathbf{T}}}(G)$, this shows that $\phi_{\mathbf{T}}$ is an algebra homomorphism.

The last assertion follows from the existence of bounded approximate identities in $L_{\omega_{\mathrm{T}}}^{1}(G)$, see for example [7], theorem 3.3.23 or [28], section 5.1.9, as indicated in [2], but we give the details for the sake of completeness. Let $K$ be a compact neighbourhood of $0_{G}$. For every open set $U \subset K$ containing $0_{G}$ set $f_{U}(g)=m(U)^{-1}$ if $g \in U, f_{U}(g)=0$ otherwise, so that $\int_{G} f_{U}(g) d m(g)=1$. We have, for $x \in X, l \in X_{*}$,

$$
\begin{aligned}
\left|\left\langle\phi_{\mathbf{T}}\left(f_{U}\right) x-x, l\right\rangle\right| & =\left|\int_{U}\langle T(g) x, l\rangle f_{U}(g) d m(g)-\langle x, l\rangle \int_{U} f_{U}(g) d m(g)\right| \\
& \leq \sup _{g \in U}\left|\left\langle T(g) x-T\left(0_{G}\right) x, l\right\rangle\right| \int_{U} f_{U}(g) d m(g) \\
& =\sup _{g \in U}\left|\left\langle T(g) x-T\left(0_{G}\right) x, l\right\rangle\right|
\end{aligned}
$$

and so there exists a sequence $\left(U_{n}\right)_{n \geq 1}$ of open subsets of $K$ containing $0_{G}$ such that $\lim _{n \rightarrow+\infty}\left\langle\phi_{\mathbf{T}}\left(f_{U_{n}}\right) x-x, l\right\rangle=0$, since the representation is weakly continuous. 


\section{A general spectral mapping theorem}

Let $\mathcal{A}$ be a Banach algebra, and let $\rho(a):=\lim _{n \rightarrow+\infty}\left\|a^{n}\right\|^{\frac{1}{n}}$ be the spectral radius of $a \in \mathcal{A}$, so that $\rho(a)=\sup _{\chi \in \widehat{\mathcal{A}} \cup\{0\}}|\chi(a)|$ if $\mathcal{A}$ is commutative, where $\widehat{A}$ denotes the space of characters of $A$, endowed with the Gelfand topology. A commutative Banach algebra $\mathcal{A}$ is said to be radical if $\rho(a)=0$ for every $a \in \mathcal{A}$, which means that $\widehat{\mathcal{A}}=\emptyset$. In the other direction a commutative Banach algebra $\mathcal{A} \neq\{0\}$ is said to be semisimple if $\cap_{\chi \in \widehat{\mathcal{A}}} k e r(\chi)=\{0\}$, which means that $\rho(a)>0$ for every $a \in \mathcal{A} \backslash\{0\}$. It follows from Shilov's idempotent theorem [7], theorem 2.4.33 that if $\mathcal{A}$ is semisimple and $\widehat{\mathcal{A}}$ is compact, then $\mathcal{A}$ is unital. If $\mathcal{A}$ is unital and $a \in \mathcal{A}$, $\operatorname{spec}(a)$ denotes the spectrum of $a$.

Let $\mathcal{A}$ be a commutative Banach algebra, and let $S \subset \mathcal{A}$. The hull of $S$ is defined by the formula

$$
h_{\mathcal{A}}(S):=\{\chi \in \widehat{\mathcal{A}}: \chi(a)=0 \forall a \in S\},
$$

and we have $h(S)=h(\overline{\mathcal{I}(S)})$, where $\mathcal{I}(S)$ denotes the ideal of $\mathcal{A}$ generated by $S$. We will often write $h(S)$ instead of $h_{\mathcal{A}}(S)$ if there is no risk of confusion. If $\mathcal{A}$ is not radical, the Gelfand transform $\widehat{a} \in \mathcal{C}(\widehat{\mathcal{A}})$ of $a \in \mathcal{A}$ is defined by the formula

$$
\widehat{a}(\chi):=\chi(a) \quad(\chi \in \widehat{\mathcal{A}}) .
$$

We now introduce the classical notions of regularity and spectral synthesis.

Definition 3.1. A non radical commutative Banach algebra $\mathcal{A}$ is said to be regular if for every proper closed subset $F$ of $\widehat{\mathcal{A}}$ and every $\chi_{0} \in \widehat{\mathcal{A}} \backslash F$ there exists $a \in \mathcal{A}$ such that $\chi_{0}(a)=1$ and $\chi(a)=0$ for every $\chi \in F$.

$A$ commutative non unital Banach algebra $\mathcal{A}$ is said to satisfy spectral synthesis if $h(\mathcal{I}) \neq \emptyset$ for every proper closed ideal $\mathcal{I}$ of $\mathcal{A}$.

We list below some standard properties of a commutative semisimple regular Banach algebra $\mathcal{A}$.

1. $\mathcal{A}$ is normal: for every closed subset $F$ of $\widehat{\mathcal{A}}$ and every compact subset $K$ of $\widehat{\mathcal{A}}$ disjoint from $F$ there exists $a \in \mathcal{A}$ such that $\chi(a)=0$ for every $a \in F$ and $\chi(a)=1$ for every $\chi \in K$, see for example [7], proposition 4.1.14.

2. Let $F \subset \widehat{\mathcal{A}}$ be closed and nonempty, set $\mathcal{I}_{F}:=\{a \in \mathcal{A}: \chi(a)=0 \forall \chi \in F\}$ and denote by $\mathcal{J}_{F}$ the set of all $a \in \mathcal{A}$ such that there exists an open subset $U_{a} \supset F$ of $\widehat{\mathcal{A}}$ satisfying $\chi(a)=0$ for every $\chi \in U_{a}$. Then $h\left(\mathcal{J}_{F}\right)=h\left(\mathcal{I}_{F}\right)=F$, and every ideal $\mathcal{I}$ of $\mathcal{A}$ such that $h(\mathcal{I})=F$ satisfies $\mathcal{J}_{F} \subset \mathcal{I} \subset \mathcal{I}_{F}$, see for example [28], proposition 3.2.6 or 7.3.2. In particular, if $\mathcal{A}$ is not unital and $h(\mathcal{I})=\emptyset$, then $a \in \mathcal{I}$ for every $a \in \mathcal{A}$ such that $\widehat{a}$ is supported by some compact subset of $\widehat{\mathcal{A}}$.

3. Let $\left(\mathcal{I}_{\lambda}\right)_{\lambda \in \Lambda}$ be a family of closed ideals of $\mathcal{A}$. Then

$$
\cup\left\{h\left(\mathcal{I}_{\lambda}\right): \lambda \in \Lambda\right\} \text { is dense in } h\left(\cap\left\{\mathcal{I}_{\lambda}: \lambda \in \Lambda\right\}\right) .
$$


4. Let $\phi$ be a homomorphism from $\mathcal{A}$ into a commutative unital Banach algebra $\mathcal{B}$ of unit element $e_{\mathcal{B}}$. Set $\phi^{*}(\chi)=\chi \circ \phi$ for $\chi \in \widehat{\mathcal{B}}$. Then we have, see for example [7], proposition 4.1.27.,

$$
\begin{gathered}
h(\operatorname{ker}(\phi)) \subset \phi^{*}(\widehat{\mathcal{B}}) \subset h(k e r(\phi)) \cup\{0\}, \\
\widehat{a}\left(h(k \operatorname{ker}(\phi)) \subset \operatorname{spec}_{\mathcal{B}}(\phi(a)) \subset \widehat{a}(h(k e r(\phi)) \cup\{0\} \quad(a \in \mathcal{A}) .\right.
\end{gathered}
$$

If $e_{\mathcal{B}} \in \overline{\phi(\mathcal{A})}$, then $e_{\mathcal{B}} \in \phi(\mathcal{A}), h(k e r(\phi))=\phi^{*}(\widehat{\mathcal{B}})$ is compact, and we have

$$
\operatorname{spec}_{\mathcal{B}}(\phi(a))=\widehat{a}(h(k e r(\phi)) \quad(a \in \mathcal{A}) .
$$

Conversely if $h(k e r(\phi))$ is compact and nonempty, then $\phi(\mathcal{A})$ is unital. Moreover $\phi^{*}$ is a homeomorphism from $\widehat{\mathcal{B}}$ onto $h(\operatorname{ker}(\phi))$ if $\phi(\mathcal{A})$ is dense in $\mathcal{B}$.

Property 3 is well-known and easy to prove: denote $F$ the closure of $\cup\left\{h\left(\mathcal{I}_{\lambda}\right)\right.$ : $\lambda \in \Lambda\}$, so that $F \subset h\left(\cap\left\{\mathcal{I}_{\lambda}: \lambda \in \Lambda\right\}\right)$. If $\chi \notin F$, let $U \subset \widehat{\mathcal{A}}$ be an open set such that $F \subset U$ and $\chi \notin \bar{U}$. There exists $a \in \mathcal{A}$ such that $\hat{a}(\chi)=1$ and $\hat{a}(\bar{U})=\{0\}$. Since $h\left(\mathcal{I}_{\lambda}\right) \subset U$, we have $a \in \mathcal{I}_{\lambda}$ for $\lambda \in \Lambda$, and $\chi \notin h\left(\cap\left\{\mathcal{I}_{\lambda}: \lambda \in \Lambda\right\}\right)$.

Property 4 means that $\phi(\mathcal{A})$ is a "full subalgebra" of $\mathcal{B}$ if $\mathcal{A}$ is semisimple and if $\overline{\phi(\mathcal{A})}$, or, equivalently, $\mathcal{A}$, contains the unit element of $\mathcal{B}$, since in this case $\operatorname{inv}(\phi(\mathcal{A}))=\operatorname{inv}(\mathcal{B}) \cap \phi(\mathcal{A})($ inv denotes the set of invertible elements). Since the group $\mathcal{G}$ of invertible elements of a unital Banach algebra is open, and since the map $x \mapsto x^{-1}$ is continuous on $\mathcal{G}$, this shows that in this situation the Banach algebra $\overline{\phi(\mathcal{A})}$ is also a full subalgebra of $\mathcal{B}$ (this property also follows from the regularity of $\overline{\phi(\mathcal{A})}$, see [19], lemma 1$)$.

The fact that $e_{\mathcal{B}} \in \overline{\phi(\mathcal{A})}$ implies that $e_{\mathcal{B}} \in \phi(\mathcal{A})$ when $\mathcal{A}$ is semisimple and regular is also standard. In this situation $h(k e r(\phi))$ is compact and nonempty. Let $U$ be a compact subset of $\widehat{\mathcal{A}}$ the interior of which contains $h(\operatorname{ker}(\phi))$, and let $u \in \mathcal{A}$ such that $\widehat{u}(U)=\{1\}$. Then $a-a u \in \mathcal{J}_{h(\operatorname{ker}(\phi))} \subset \operatorname{ker}(\phi)$ for every $a \in \mathcal{A}$. So $\phi(u)$ is a unit element of $\phi(\mathcal{A})$, and $\phi(u)=e_{\mathcal{B}}$.

Notice that it may happen that $e_{\mathcal{B}} \in \overline{\phi(\mathcal{A})}$ and that $\phi(\mathcal{A})$ is not unital when $\phi$ is a homomorphism from a commutative semisimple Banach algebra $\mathcal{A}$ into a commutative unital Banach algebra: for $r>0$ denote by $\mathcal{A}_{r}$ the Banach algebra of holomorphic functions on the open disc $D_{r}:=D(0, r)$ which admit an holomorphic extension to the closed disc $\overline{D_{r}}$. Set $\mathcal{M}_{1}:=\left\{f \in \mathcal{A}_{1}: f(1)=0\right\}$. For $f \in \mathcal{M}_{1}$ denote by $\phi(f)$ the restriction of $f$ to the closed disc $\overline{D_{1 / 2}}$. Set $e_{n}(z)=\frac{z-1}{z-1-1 / n}$ for $|z| \leq 1$. Then $e_{n} \in \mathcal{M}_{1}, \lim _{n \rightarrow+\infty} \sup _{|z| \leq 1 / 2}\left|1-e_{n}(z)\right|=0$, an easy verification shows that $\phi\left(\mathcal{M}_{1}\right)$ is dense in the unital Banach algebra $\mathcal{A}_{1 / 2}$, but $\phi\left(\mathcal{M}_{1}\right)$ does not contain 1 and $\phi\left(e_{n}\right)^{-1} \notin \phi\left(\mathcal{M}_{1}\right)$ for $n \geq 1$.

The closed subalgebra $\operatorname{reg}(\mathcal{A})$ of a commutative Banach algebra generated by the union of all closed regular subalgebras of $\mathcal{A}$ is itself a closed regular subalgebra of $\mathcal{A}$, called the maximal regular subalgebra of $\mathcal{A}$. This result goes back to Albrecht [1] in the semisimple case, see also the proof of [27] given in [7], proposition 4.1.17. A very simple proof of this fact based on the hull-kernel topology was obtained by 
Inoue and Takahasi in [18], see [28], corollary 3.2.11. Notice that $\operatorname{reg}(\mathcal{A})$ is unital if $\mathcal{A}$ has a unit element $e$, since $\mathbb{C} e$ is regular.

We now state an abstract version of the spectral mapping theorem. We will use below the fact that if $\mathcal{I}$ is a closed ideal of $\mathcal{A}$ not contained in the radical of $\mathcal{A}$, then every $\chi \in \widehat{\mathcal{I}}$ has a unique extension $\tilde{\chi}$ to $\mathcal{A}$ given by the formula $\tilde{\chi}(a):=\chi(a u)$, where $u$ is any element of $\mathcal{I}$ such that $\chi(u)=1$. The map $\chi \mapsto \tilde{\chi}$ is clearly an homeomorphism from $\widehat{\mathcal{I}}$ onto $\widehat{\mathcal{A}} \backslash h(\mathcal{I})$.

Theorem 3.2. Let $\mathcal{A}$ be a commutative unital Banach algebra, let $X$ be a Banach space, let $X_{*}$ be a subspace of $X^{\prime}$ such that $\sup _{l \in X_{*},\|l\| \leq 1}|\langle x, l\rangle|=\|x\|$ for $x \in X$, and let $\phi: \mathcal{A} \rightarrow \mathcal{B}(X)$ be a continuous unital homomorphism. Let $\mathcal{I}$ be a closed ideal of $\mathcal{A}$, and for $\chi \in \widehat{\mathcal{I}}$ denote by $\tilde{\chi}$ the unique extension of $\chi$ to $\mathcal{A}$.

Assume that $\mathcal{I}$ is a semisimple regular Banach algebra which satisfies spectral synthesis, and that we have

$$
\inf _{a \in \mathcal{I}}|\langle\phi(a) x-x, l\rangle|=0 \quad\left(x \in X, l \in X_{*}\right) .
$$

Then the following properties hold:

(i) The set $h_{\mathcal{I}}(\operatorname{ker}(\phi) \cap \mathcal{I})$ is not empty.

(ii) If $h_{\mathcal{I}}(k e r(\phi) \cap \mathcal{I})$ is compact, then $I \in \phi(\mathcal{I}), \phi(\mathcal{I})=\phi(\mathcal{A}), h(k e r(\phi))=\{\tilde{\chi}$ : $\left.\chi \in h_{\mathcal{I}}(\operatorname{ker}(\phi) \cap \mathcal{I})\right\}$, and

$$
\operatorname{spec}(\phi(a))=\left\{\tilde{\chi}(a): \chi \in h_{\mathcal{I}}(k e r(\phi) \cap \mathcal{I})\right\} \quad(a \in \mathcal{A}) .
$$

(iii) If $h_{\mathcal{I}}(k e r(\phi) \cap \mathcal{I})$ is not compact, then the weak*-closure of $h_{\mathcal{I}}(k e r(\phi) \cap \mathcal{I})$ in the unit ball of the dual of $\mathcal{I}$ contains $0,0 \in \overline{\widehat{a}\left(h_{\mathcal{I}}(k e r(\phi) \cap \mathcal{I})\right)}$, and $\operatorname{spec}(\phi(a)) \backslash\{0\} \subset \widehat{a}\left(h_{\mathcal{I}}(k e r(\phi) \cap \mathcal{I})\right)$ for $a \in \mathcal{I}$.

(iv) If $\mathcal{U}$ is a closed regular subalgebra of $\mathcal{A}$ containing $\mathcal{I}$, then the set $\left\{\tilde{\chi}_{\mid \mathcal{U}}\right.$ : $\left.\chi \in h_{\mathcal{I}}(k e r(\phi) \cap \mathcal{I})\right\}$ is dense in $h_{\mathcal{U}}(\operatorname{ker}(\phi) \cap \mathcal{I})$, and the set $\{\tilde{\chi}(\phi(a)): \chi \in$ $\left.h_{\mathcal{I}}(k e r(\phi) \cap \mathcal{I})\right\}$ is dense in spec $(\phi(a))$ for every $a \in \mathcal{U}$.

Proof: (i) Let $x \in X \backslash\{0\}$ and $l \in X_{*}$ be such that $\langle x, l\rangle \neq 0$. It follows from (3.5) that $\langle\phi(a) x, l\rangle \neq 0$ for some $a \in \mathcal{I}, \operatorname{ker}(\phi) \cap \mathcal{I}$ is a proper closed ideal of $\mathcal{I}$ and $h_{\mathcal{I}}(\operatorname{ker}(\phi) \cap \mathcal{I}) \neq \emptyset$.

(ii) If $h_{\mathcal{I}}(\operatorname{ker}(\phi) \cap \mathcal{I})$ is compact, then $\phi(\mathcal{I})$ is unital and it follows from $(3.5)$ that $I \in \phi(\mathcal{I})$, and so $\phi(\mathcal{I})=\phi(\mathcal{A})$ since $\phi(\mathcal{I})$ is an ideal of $\phi(\mathcal{A})$. Let $u \in \mathcal{I}$ such that $e-u \in \operatorname{ker}(\phi)$, where $e$ denotes the unit element of $\mathcal{A}$. Then $\chi(u)=\chi(e)=1$ for $\chi \in h(k e r(\phi))$, and $\chi \notin h(\mathcal{I})$. Hence $h(\operatorname{ker}(\phi))=\left\{\tilde{\chi}: \chi \in h_{\mathcal{I}}(\operatorname{ker}(\phi) \cap \mathcal{I})\right\}$, since $\tilde{\chi} \in h(k e r(\phi))$ for every $\chi \in h_{\mathcal{I}}(k e r(\phi) \cap \mathcal{I})$. Let $\mathcal{B}$ be a maximal commutative subalgebra of $\mathcal{B}(X)$ containing $\phi(\mathcal{A})$. Then it follows from $(3.4)$ applied to $\mathcal{B}$ that $\operatorname{spec}(\phi(a))=\operatorname{spec}(\phi(a u))=\operatorname{spec}_{\mathcal{B}}(\phi(a u))=\left\{\chi(a u): \chi \in h_{\mathcal{I}}(k e r(\phi) \cap \mathcal{I})\right\}=$ $\left\{\tilde{\chi}(a): \chi \in h_{\mathcal{I}}(k e r(\phi) \cap \mathcal{I})\right\}$.

(iii) Now assume that $h_{\mathcal{I}}(k e r(\phi) \cap \mathcal{I})$ is not compact. Then $I \notin \phi(\mathcal{I}), 0 \in$ $\overline{h_{\mathcal{I}}(\operatorname{ker}(\phi) \cap \mathcal{I})}$, and $0 \in \overline{\widehat{a}\left(h_{\mathcal{I}}(\operatorname{ker}(\phi) \cap \mathcal{I})\right)}$. The fact that $\operatorname{spec}(\phi(a)) \backslash\{0\} \subset$ $\widehat{a}\left(h_{\mathcal{I}}(\operatorname{ker}(\phi) \cap \mathcal{I})\right)$ for $a \in \mathcal{I}$ follows from (3.2) applied to $\mathcal{B}$. 
(iv) Denote by $\mathcal{K}(\widehat{\mathcal{I}})$ the set of all nonempty compact subsets of $\widehat{\mathcal{I}}$, and for $K \in \mathcal{K}(\widehat{\mathcal{I}})$ denote by $\mathcal{I}_{K}$ the set of all $a \in \mathcal{I}$ such that $\chi(a)=0$ for every $\chi \in K$. Then $\mathcal{I}_{K}$ is a closed ideal of $\mathcal{A}$. Set

$$
X_{K}=\left\{x \in X: \phi(a) x=0 \forall a \in \mathcal{I}_{K}\right\} .
$$

Then $X_{K}$ is a closed subspace of $X$, and $\phi(a)\left(X_{K}\right) \subset X_{K}$ for every $a \in \mathcal{A}$. Set $\phi_{K}(a)=\phi(a)_{\left.\right|_{X_{K}}}$ for $a \in \mathcal{A}$. Then $\phi_{K}: \mathcal{A} \rightarrow \mathcal{B}\left(X_{K}\right)$ is a unital homomorphism.

Let $\chi_{0} \in \widehat{\mathcal{I}} \backslash K$, and let $u \in \mathcal{I}$ be such that $\chi_{0}(u)=1$ and $\chi(u)=0$ for every $\chi \in K$. Then $u \in \mathcal{I}_{K} \subset \operatorname{ker}\left(\phi_{K}\right)$, and $\chi_{0} \notin h_{\mathcal{I}}\left(k e r\left(\phi_{K}\right) \cap \mathcal{I}\right)$. This shows that $h_{\mathcal{I}}\left(\operatorname{ker}\left(\phi_{K}\right) \cap \mathcal{I}\right) \subset K$ is compact. It follows then from that $h\left(\operatorname{ker}\left(\phi_{K}\right)\right)=\{\tilde{\chi}$ : $\left.\chi \in h\left(\operatorname{ker}\left(\phi_{K}\right) \cap \mathcal{I}\right)\right\}$.

Let $\mathcal{U}$ be a closed regular subalgebra of $\mathcal{A}$ containing $\mathcal{I}$. We have that $h_{\mathcal{U}}\left(\operatorname{ker}\left(\phi_{K}\right) \cap \mathcal{U}\right)=\left\{\tilde{\chi}_{\mid \mathcal{U}}: \chi \in h_{\mathcal{I}}\left(\operatorname{ker}\left(\phi_{K}\right) \cap \mathcal{I}\right)\right\} \subset\left\{\tilde{\chi}_{\mid \mathcal{U}}: \chi \in h_{\mathcal{I}}(\operatorname{ker}(\phi) \cap \mathcal{I})\right\}$.

Clearly, $\operatorname{ker}(\phi) \subset \cap\left\{\operatorname{ker}\left(\phi_{K}\right): K \in \mathcal{K}(\widehat{\mathcal{I}})\right\}$. Conversely assume that $a \in \mathcal{A}$ and that $\phi_{K}(a)=0$ for every $K \in \mathcal{K}(\widehat{\mathcal{I}})$. Set $\Delta_{K}=\mathcal{I}_{\widehat{\mathcal{I}} \backslash \AA}$, where $\stackrel{\circ}{K}$ denotes the interior of $K \in \mathcal{K}(\widehat{\mathcal{I}})$. Let $x \in X$, and let $l \in X_{*}$. Since $\mathcal{I}$ satisfies spectral synthesis, $\cup\left\{\Delta_{K}: K \in \mathcal{K}(\widehat{\mathcal{I}})\right\}$ is dense in $\mathcal{I}$, and there exists a sequence $\left(u_{n}\right)_{n \geq 1}$ of elements of $\cup\left\{\Delta_{K}: K \in \mathcal{K}(\widehat{\mathcal{I}})\right\}$ such that $\langle\phi(a) x, l\rangle=\lim _{n \rightarrow+\infty}\left\langle\phi\left(u_{n}\right) \phi(a) x, l\right\rangle=$ $\lim _{n \rightarrow+\infty}\left\langle\phi(a) \phi\left(u_{n}\right) x, l\right\rangle$. Let $K_{n} \in \mathcal{K}(\widehat{\mathcal{I}})$ such that $u_{n} \in \Delta_{K_{n}}$. If $b \in \mathcal{I}_{K_{n}}$, then $b u_{n} \in \cap_{\chi \in \widehat{I}} \operatorname{ker}(\chi)=\{0\}$, and so $\phi\left(u_{n}\right) x \in X_{K_{n}}$ and $\phi(a) \phi\left(u_{n}\right) x=0$. Hence $\langle\phi(a) x, l\rangle=0$ and $a \in \operatorname{ker}(\phi)$.

Hence $\operatorname{ker}(\phi)=\cap\left\{k \operatorname{ker}\left(\phi_{K}\right): K \in \mathcal{K}(\widehat{\mathcal{I}})\right\}, \operatorname{ker}(\phi) \cap \mathcal{U}=\cap\left\{\operatorname{ker}\left(\phi_{K}\right) \cap \mathcal{U}: K \in\right.$ $\mathcal{K}(\widehat{\mathcal{I}})\}$, and it follows from $(3.1)$ that $\left\{\tilde{\chi}_{\mid \mathcal{U}}: \chi \in h_{\mathcal{I}}(\operatorname{ker}(\phi) \cap \mathcal{I})\right\} \supset \cup\left\{h_{\mathcal{U}}\left(\operatorname{ker}\left(\phi_{K}\right) \cap\right.\right.$ $\mathcal{U}): K \in \mathcal{K}(\widehat{\mathcal{I}})\}$ is dense in $h_{\mathcal{U}}(k e r(\phi) \cap \mathcal{U})$.

It follows then from $(3.4)$ that the set $\left\{\tilde{\chi}(a): \chi \in h_{\mathcal{I}}(\operatorname{ker}(\phi) \cap \mathcal{I})\right\}$ is dense in $\operatorname{spec}(\phi(a))$ for every $a \in \mathcal{U}$.

\section{The weak spectral mapping theorem for representations of locally compact abelian groups}

Consider again a locally compact abelian group $G$. A submultiplicative locally bounded measurable weight on $G$ is a function $\omega: G \rightarrow(0,+\infty)$ which is measurable with respect to the Haar measure $m$ on $G$ and satisfies $\omega\left(g_{1}+g_{2}\right) \leq$ $\omega\left(g_{1}\right) \omega\left(g_{2}\right)$ for $g_{1}, g_{2} \in G$ and $\sup _{g \in K} \omega(g)<+\infty$ for every compact subset $K$ of $G$. In this situation the space $L_{\omega}^{1}(G)$ of all Haar measurable functions $f$ on $G$ satisfying $\|f\|_{\omega}:=\int_{G}|f(g)| \omega(g) d m(g)<+\infty$ is a Banach algebra with respect to convolution. If $\lim _{n \rightarrow+\infty} \omega(n g)^{\frac{1}{n}}=1$ for every $g \in G$, then $\omega(g) \geq 1$ for every $g \in G$, and the map $s \mapsto \chi_{s}$ is a homeomorphism from the dual group $\widehat{G}$ onto $\widehat{L_{\omega}^{1}(G)}$, where the character $\chi_{s}$ is defined by the formula 


$$
\chi_{s}(f)=\int_{G} f(g)\langle g, s\rangle d m(g)=\widehat{f}(s) \quad\left(f \in L_{\omega}^{1}(G)\right) .
$$

Definition 4.1. A submultiplicative measurable locally bounded weight on $G$ is said to be nonquasianalytic when it satisfies the condition

$$
\sum_{n=1}^{+\infty} \frac{\log (\omega(n g))}{1+n^{2}}<+\infty \quad(g \in G) .
$$

Notice that if $\omega$ is any submultiplicative weight on $G$ then we have $\omega(g) \geq$ $\lim _{n \rightarrow+\infty} \omega(n g)^{\frac{1}{n}}$ for $g \in G$. Condition (4.2) implies that $\lim _{n \rightarrow+\infty} \omega(n g)^{\frac{1}{n}} \leq 1$ for every $g \in G$. Since $\lim _{n \rightarrow+\infty} \omega(n g)^{\frac{1}{n}} \cdot \lim _{n \rightarrow+\infty} \omega(-n g)^{\frac{1}{n}} \geq 1$, we have in fact $\omega(g) \geq \lim _{n \rightarrow+\infty} \omega(n g)^{\frac{1}{n}}=1$ for $g \in G$ if $\omega$ is nonquasianalytic. This allows to identify the character space $\widehat{L_{\omega}^{1}(G)}$ with the dual group $\widehat{G}$ by using formula (4.1).

The following result goes back to Domar [8].

Theorem 4.2. [8] Let $G$ be a locally compact abelian group, let $\omega$ be a submultiplicative measurable locally bounded nonquasianalytic weight on $G$. Then the convolution algebra $L_{\omega}^{1}(G)$ is a regular Banach algebra which satisfies spectral synthesis.

We now state a general version of the weak spectral mapping theorem.

Theorem 4.3. Let $G$ be a locally compact abelian group, let $\left(X, X_{*}\right)$ be a dual pairing and let $\mathbf{T}=(T(g))_{g \in G}$ be a representation of $G$ on $X$ which is weakly continuous with respect to $\left(X, X_{*}\right)$. Assume that the representation satisfies the condition

$$
\sum_{n=1}^{+\infty} \frac{\log \|T(n g)\|}{1+n^{2}}<+\infty \quad(g \in G) .
$$

Set $\mathcal{M}_{\omega_{\mathbf{T}}}(G)=\left\{\mu \in \mathcal{M}(G): \int_{G}^{*}\|T(g)\| d|\mu|(g)<+\infty\right\}$, and let $\phi_{\mathbf{T}}: \mathcal{M}_{\omega_{\mathbf{T}}}(G) \rightarrow$ $\mathcal{B}(X)$ be the unital homomorphism defined by the formula

$$
\left\langle\phi_{\mathbf{T}}(\mu) x, l\right\rangle=\int_{G}\langle T(g) x, l\rangle d \mu(g) \quad\left(\mu \in \mathcal{M}_{\omega_{\mathbf{T}}}(G), x \in X, l \in X_{*}\right) .
$$

Let

$$
\operatorname{spec}(\mathbf{T}):=\left\{s \in \widehat{G}: \hat{f}(s)=0 \forall f \in \operatorname{ker}\left(\phi_{\mathbf{T}}\right) \cap L_{\omega_{\mathbf{T}}}^{1}(G)\right\}
$$

be the Arveson spectrum of the representation. Then the following properties hold.

(i) $\operatorname{spec}(\mathbf{T})$ is nonempty.

(ii) If $\operatorname{spec}(\mathbf{T})$ is compact, then the representation is continuous with respect to the norm of $\mathcal{B}(X), \phi_{\mathbf{T}}\left(\mathcal{M}_{\omega_{\mathbf{T}}}(G)\right)=\phi_{\mathbf{T}}\left(L_{\omega_{\mathbf{T}}}^{1}(G)\right)$ and $\operatorname{spec}\left(\phi_{\mathbf{T}}(\mu)\right)=$ $\hat{\mu}(\operatorname{spec}(\mathbf{T}))$ for every $\mu \in \mathcal{M}_{\omega_{\mathbf{T}}}(G)$.

(iii) If $\mu$ is contained in a regular subalgebra of $\mathcal{M}_{\omega_{\mathbf{T}}}(G)$, then the set $\hat{\mu}(\operatorname{spec}(\mathbf{T}))$ is dense in spec $\left(\phi_{\mathbf{T}}(\mu)\right)$. In particular the set $\{\langle g, s\rangle: s \in \operatorname{spec}(\mathbf{T})\}$ is dense in $\operatorname{spec}(T(g))$ for every $g \in G$. 
Proof: It follows from proposition 2.4 that $\inf _{f \in L_{\omega_{\mathbf{T}}}^{1}(G)}\left|\left\langle\phi_{\mathbf{T}}(f) x-x, l\right\rangle\right|=0$, and it follows from theorem 4.2 that the convolution algebra $L_{\omega_{\mathbf{T}}}^{1}(G)$ is regular and satisfies spectral synthesis. Denote by $\delta_{g}$ the Dirac measure at $g \in G$. Then $T(g)=$ $\phi_{\mathbf{T}}\left(\delta_{g}\right)$. Since the discrete topology on $G$ is locally compact, the convolution algebra $l_{\omega_{\mathbf{T}}}^{1}(G)=\overline{\operatorname{span}\left\{\delta_{g}\right\}_{g \in G}}$ is also regular, and it follows from theorem 2.4 that (i), (iii) and the last two assertions of $(i i)$ hold. Since the map $g \mapsto f * \delta_{g}$ is continuous on $G$ with respect to the norm of $L^{1}(G)$ and since $\phi_{\mathbf{T}}$ is continuous, a density argument shows that the map $g \mapsto \phi_{\mathbf{T}}(f) T(g)$ is continuous with respect to the norm of $\mathcal{B}(X)$ for every $f \in L_{\omega_{\mathbf{T}}}^{1}(G)$. Hence the representation is continuous with respect to the norm of $\mathcal{B}(X)$ if $\operatorname{spec}(\mathbf{T})$ is compact, since in this case $I=\phi_{\mathbf{T}}(f)$ for some $f \in L_{\omega_{\mathbf{T}}}^{1}(G)$.

Notice that if $\tau$ is a locally compact group topology on $G$ coarser than the given one, then $L_{\omega_{\mathbf{T}}}^{1}\left(G, m_{\tau}\right)$ is a regular subalgebra of $\mathcal{M}_{\omega_{\mathbf{T}}}(G)$, and so $\hat{\mu}(\operatorname{spec}(\mathbf{T}))$ is dense in $\operatorname{spec}\left(\phi_{\mathbf{T}}(\mu)\right)$ for $\mu \in L_{\omega_{\mathbf{T}}}^{1}\left(G, m_{\tau}\right)$ if $\mathbf{T}$ is non-quasianalytic. The union of all these convolution algebras may be strictly contained in $\operatorname{reg}\left(\mathcal{M}_{\omega_{\mathbf{T}}}(G)\right)$, see [17].

\section{Link between the Arveson spectrum and the spectrum of the generator of a $\mathcal{C}_{0}$-group}

Consider the case $G=\mathbb{R}, X_{*}=X^{\prime}, T$ a $\mathcal{C}_{0}$-group on $X$ with non-quasianalytic weight. Denote by $A$ the infinitesimal generator of $T$; this is an unbounded linear operator on $X$ with dense domain $\mathcal{D}_{A}$. We identify $\widehat{G}$ with $i \mathbb{R}$ and for $f \in L_{\omega_{\mathbf{T}}}^{1}(\mathbb{R})$ the Fourier transform is

$$
\widehat{f}(i s)=\int_{\mathbb{R}} f(t) e^{i t s} d t=\chi_{i s}(f) .
$$

For the convenience of the reader, we give a proof of the following well-known result (stated in a slightly less general form in corollary 4.1 of [32]).

\section{Theorem 5.1.}

$$
\operatorname{spec}(\mathbf{T})=\sigma(A)
$$

Proof: For the inclusion "つ" we use the following two poperties:

i) For $f \in L_{\omega_{\mathbf{T}}}^{1}(\mathbb{R})$ with $\phi_{\mathbf{T}}(f)=0$ we have $\sigma_{a p}(A) \subset k e r \widehat{f}\left(\sigma_{a p}\right.$ is the approximate point spectrum of the operator).

ii) $\sigma(A)=\sigma_{a p}(A)$

Proof of the inclusion " $\supset$ ":

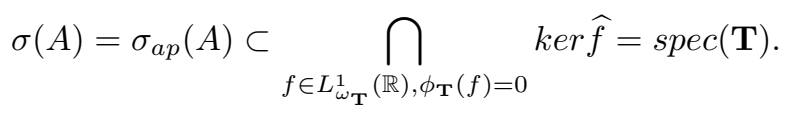


0 . Then

Proof of i): Let $\lambda \in \sigma_{a p}(A), x_{n} \in \mathcal{D}_{A}$ a sequence with $\left\|x_{n}\right\|=1, \lambda x_{n}-A x_{n} \rightarrow$

$$
e^{\lambda t} x_{n}-T(t) x_{n}=\int_{0}^{t} e^{\lambda(t-s)} T(s)\left(\lambda x_{n}-A x_{n}\right) d s
$$

and $e^{\lambda t} x_{n}-T(t) x_{n} \rightarrow 0$. We compute

$$
\begin{aligned}
\left\|\phi_{\mathbf{T}}(f)\right\| & =\sup _{\|x\|=1}\left\|\phi_{\mathbf{T}}(f) x\right\| \\
& \geq \varlimsup \lim \left\|\phi_{\mathbf{T}}(f) x_{n}\right\| \\
& =\varlimsup \lim \left\|\int_{-\infty}^{\infty}\left(T(t) x_{n}-e^{\lambda t} x_{n}+e^{\lambda t} x_{n}\right) f(t) d t\right\| \\
& \geq \varlimsup \lim \left|\int_{-\infty}^{\infty} e^{\lambda t} f(t) d t\right|\left\|x_{n}\right\|-\varlimsup \\
& =\mid \widehat{\lim } \| \int_{-\infty}^{\infty}(T(t) \mid .
\end{aligned}
$$

So $\phi_{\mathbf{T}}(f)=0$ implies $\widehat{f}(\lambda)=0$.

Proof of ii): This follows from the non-quasianalyticity of the weight, since $\sigma(A) \subset i \mathbb{R}$, and therefore $\sigma(A)=\partial \sigma(A)$, the boundary.

For the opposite inclusion " $\subset$ " we use the following two properties:

iii) For $f \in L_{\omega_{\mathbf{T}}}^{1}(\mathbb{R})$ such that $\widehat{f}$ has compact support and vanishes on an open set containing $\sigma(A)$ we have $\phi_{\mathbf{T}}(f)=0$.

iv) $L_{\omega_{\mathbf{T}}}^{1}(\mathbb{R})$ is a regular Banach algebra.

Proof of the inclusion " $\subset$ " : If $\lambda \in i \mathbb{R} \backslash \sigma(A)$, choose first a closed neighbourhood $U$ of $\sigma(A)$ (in the euclidean topology) such that $\lambda \notin U$. Then, by regularity, there exists $f \in L_{\omega_{\mathbf{T}}}^{1}(\mathbb{R})$ such that $\widehat{f}=0$ on $U$ and $\widehat{f}(\lambda)=1$, and there exists $g \in L_{\omega_{\mathbf{T}}}^{1}(\mathbb{R})$ such that $\widehat{g}$ has compact support and $\widehat{g}(\lambda)=1$. Set $h=f * g$. Then by iii) we have $\phi_{\mathbf{T}}(h)=0$, with $\widehat{h}(\lambda)=1$. Hence $\lambda \notin \operatorname{spec}(\mathbf{T})$.

Proof of iii): By Lebesgue and by inverse Fourier transform theorems we have

$$
\phi_{\mathbf{T}}(f) x=\lim _{\delta \rightarrow 0+} \int_{-\infty}^{\infty} e^{-\delta|t|} T(t) x f(t) d t, \quad f(t)=\frac{1}{2 \pi} \int_{-\infty}^{\infty} \widehat{f}(-i s) e^{i s t} d s .
$$

Furthermore, for $\Re \lambda>0$ we have

$$
(\lambda-A)^{-1} x=\int_{0}^{\infty} e^{-\lambda t} T(t) x d t, \quad(\lambda+A)^{-1} x=\int_{-\infty}^{0} e^{\lambda t} T(t) x d t .
$$

We compute, using Fubini and Lebesgue theorems:

$$
\begin{aligned}
\phi_{\mathbf{T}}(f) x & =\frac{1}{2 \pi} \lim _{\delta \rightarrow 0+} \int_{-\infty}^{\infty}\left(\int_{-\infty}^{0}+\int_{0}^{\infty}\right) e^{i s t} e^{-\delta|t|} T(t) x d t \widehat{f}(-i s) d s \\
& =\frac{1}{2 \pi} \lim _{\delta \rightarrow 0+} \int_{-\infty}^{\infty}\left((\delta-i s-A)^{-1} x-(-\delta-i s-A)^{-1} x\right) \widehat{f}(-i s) d s \\
& =0 .
\end{aligned}
$$

Proof of iv): This follows from the non-quasianaliticity of the weight (4.3). 
The theorem is proved.

\section{References}

[1] E. Albrecht, Decomposable systems of operators in harmonic analysis, Toeplitz Centennial (I. Golberg (ed.), Birkhauser, Basel, 1982), 19-35.

[2] W. Arveson, On group of automorphisms of operator algebras, J. Funct. An. 15 (1974), 217-243.

[3] A. G. Baskakov, Inequalities of Bernshtein type in abstract harmonic analysis, Sibirskij Mat. Zhurnal 20 (1979), English translation 665-672.

[4] N. Bourbaki, Integration, Hermann, Paris, 1959.

[5] A. Connes, Une classification des facteurs de type III, Ann. scient. Éc. Norm. Sup. 4e série t. 6 (1973), 133-152.

[6] C. d'Antoni, R. Longo and L. Zsidó, A spectral mapping theorem for locally compact group algebras, Pac. J. Math. 103 (1981), 17-24.

[7] H. G. Dales, Banach algebras and automatic continuity, London Mathematical Society Monographs, vol. 24, The Clarendon Press, Oxford, 2000.

[8] Y. Domar, Harmonic analysis based on certain commutative Banach algebras, Acta Math. 96 (1956), 1-66.

[9] K.-J. Engel and R. Nagel, One-Parameter Semigroups for Linear Evolution Equations, Graduate Texts in Mathematics 194, Springer-Verlag, New-York, 2000.

[10] D. E. Evans, On the spectrum of a one-parameter strongly continuous representation, Math. Scand. 39 (1976), 80-82.

[11] E. Fašangová, A Banach algebra approach to the weak spectral mapping theorem for $C_{0}$-groups, Ulmer Seminare Heft 5 (2000), 174-181.

[12] E. Fašangová, Spectral mapping theorems and spectral space-independence, Progress Nonlin. Diff. Eqns. Appl. 55 (2003), 157-168.

[13] E. Fašangová and P. J. Miana, Spectral mapping inclusions for the Phillips functional calculus in Banach spaces and algebras, Studia Math. 167 (2005), 219-226.

[14] M. Gerlach and M. Kunze, Mean ergodic theorems on norming dual pairs. Ergodic Theory Dynam. Systems 34 (2014), 1210-1229.

[15] S.-Z. Huang, An equivalent description of non-quasianalyticity through spectral theory of $C_{0}$-groups, J. Operator Theory 33 (1994), 299-309.

[16] S.-Z. Huang, Spectral theory for non-quasianalytic representations of locally compact abelian groups, Ph.D. Thesis, Tübingen, 1995.

[17] J. Inoue, Some closed subalgebras of measure algebras and a generalization of P. J. Cohen's theorem, J. Math. Soc. Japan 23 (1971), 278-294.

[18] J. Inoue and S.-E. Takahasi, A note on the largest regular subalgebra of a Banach algebra, Proc. Amer. Math. Soc. 116 (1992), 961-962.

[19] R. Kantrowitz and M. M. Neumann, The greatest regular subalgebra of certain Banach algebras of vector-valued functions, Rend. Circ. Mat. Palermo, Ser. 2, 43 (1994), 435-446.

[20] M. Kunze, Continuity and equicontinuity of semigroups on norming dual pairs, Semigroup Forum 79 (2009), 540-560. 
[21] Y. I. Lyubich and V. I. Matsaev, Operators with separable spectrum, (Russian), Mat. Sb. (1962), 433-468.

[22] Y. I. Lyubich, V. I. Matsaev and G. M. Feldman, The separability of the spectrum of a representation of a locally compact abelian group, (Russian), translation in Dokl. Akad. Nauk SSSR 201 (1971), 1282-1284, Soviet Math. Dokl. 12 (1971), 1824-1827.

[23] Y. I. Lyubich, V. I. Matsaev and G. M. Feldman, On representations with a separable spectrum, (Russian), Funkcional. Anal. i Prilozen. 7 (1973), no. 2, 52-61.

[24] E. Marschall, A spectral mapping theorem for local multipliers, Math. Ann. 260 (1982), 143-150.

[25] E. Marschall, On the functional calculus of nonquasianalytic groups of operators and cosine functions, Rend. Circ. Mat. Palermo 35 (1986), no. 1, 58-81.

[26] R. Nagel and S.-Z. Huang, Spectral Mapping Theorems for $C_{0}$-groups satisfying Nonquasianalytic growth conditions, Math. Nach. 169 (1994), 207-218.

[27] M. M. Neumann, Commutative Banach algebras and decomposable operators, Monatsh. für Math. 113 (1992), 227-243.

[28] T. W. Palmer, Banach algebras and the general theory of $*$-algebras, Volume 1, Algebras and Banach algebras, Encyclopedia of Mathematics and its Applications, vol. 49, Cambridge University Press, 1993.

[29] H. Seferoğlu, Spectral mapping theorem for representations of measure algebras, Proc. Edinburgh Math. Soc. 40 (1997), 261-266.

[30] H. Seferoğlu, Spectral mapping theorem for Banach modules, Studia Math. 156 (2003), 99-103.

[31] H. Seferoğlu, A spectral mapping theorem for representations of one-parameter groups, Proc. Amer Math. Soc. 134 (2006), 2457-2463.

[32] H. Seferoğlu, Generators of $\mathcal{C}_{0}$-groups with smallest spectra, Math. Nachr. 280 (2007), 924-931.

[33] S.-E. Takahasi and J. Inoue, A spectral mapping theorem for some representations of compact abelian groups, Proc. Edinburgh Math. Soc. 35 (1992), 47-52.

[34] J. M. A. M. van Neerven, Elementary operator-theoretic proof of Wiener's Tauberian theorem, Rendic. Istit. Matem. Univ. Trieste., Suppl. Vol 28 (1997), 281-286.

[35] K. F. Vu and Y. I. Lyubich, On the spectral mapping theorem for one-parameter groups of operators, Zap. Nauchn. Sem. Leningrad. Otdel. Mat. Inst. Steklov (LOMI) 178 (1989), 146-150, translation in J. Soviet Math. 61 (1992), 2035-2037.

Jean Esterle

Université de Bordeaux

IMB, UMR 5251

351 , cours de la Libération

33405-Talence-Cedex

France

e-mail: esterle@math.u-bordeaux1.fr 
Eva Fašangová

Technische Universität Dresden

Fachrichtung Mathematik

Institut für Analysis

01062 Dresden

Germany

e-mail: eva.fasangova@tu-dresden.de 